\title{
Gabrb2-knockout mice displayed schizophrenia-like and comorbid phenotypes with interneuron-astrocyte-microglia dysregulation
}

Rigil K. Yeung ${ }^{1}$, Zheng-Hua Xiang ${ }^{2}$, Shui-Ying Tsang ${ }^{1}$, Rui Li $i^{1}$, Timothy Y. C. Ho ${ }^{1}$, Qi Li ${ }^{3}$, Chok-King Hui ${ }^{3}$, Pak-Chung Sham ${ }^{3}$, Ming-Qi Qiao ${ }^{4}$ and Hong Xue ${ }^{1,4}$

\begin{abstract}
Intronic polymorphisms of the GABA $A_{A}$ receptor $\beta_{2}$ subunit gene (GABRB2) under adaptive evolution were associated with schizophrenia and reduced expression, especially of the long isoform which differs in electrophysiological properties from the short isoform. The present study was directed to examining the gene dosage effects of Gabrb2 in knockout mice of both heterozygous (HT) and homozygous (KO) genotypes with respect to possible schizophrenialike and comorbid phenotypes. The KO mice, and HT mice to a lesser extent, were found to display prepulse inhibition (PPI) deficit, locomotor hyperactivity, stereotypy, sociability impairments, spatial-working and spatial-reference memory deficits, reduced depression and anxiety, and accelerated pentylenetetrazol (PTZ)-induced seizure. In addition, the KO mice were highly susceptible to audiogenic epilepsy. Some of the behavioral phenotypes showed evidence of imprinting, gender effect and amelioration by the antipsychotic risperidone, and the audiogenic epilepsy was inhibited by the antiepileptic diazepam. GABAergic parvalbumin (PV)-positive interneuron dystrophy, astrocyte dystrophy, and extensive microglia activation were observed in the frontotemporal corticolimbic regions, and reduction of newborn neurons was observed in the hippocampus by immunohistochemical staining. The neuroinflammation indicated by microglial activation was accompanied by elevated brain levels of oxidative stress marker malondialdehyde (MDA) and the pro-inflammatory cytokines tumor necrosis factor-alpha (TNF-a) and interleukin-6 (IL-6). These extensive schizophrenia-like and comorbid phenotypes brought about by Gabrb2 knockout, in conjunction with our previous findings on GABRB2 association with schizophrenia, support a pivotal role of GABRB2 in schizophrenia etiology.
\end{abstract}

Correspondence: Hong Xue (hxue@ust.hk)

${ }^{1}$ Division of Life Science, Applied Genomics Center and State Key Laboratory of Molecular Neuroscience, Hong Kong University of Science and Technology,

Clear Water Bay, Hong Kong, People's Republic of China

${ }^{2}$ Department of Neurobiology, Second Military Medical University, Shanghai, People's Republic of China

Full list of author information is available at the end of the article

These authors contributed equally: Rigil K.Yeung, Zheng-Hua Xiang, Shui-Ying Tsang.

\section{Introduction}

Schizophrenia is a multifactorial disease that results from interactions between genetic and environmental factors. The strength of genetic factors is underlined by the rise of lifetime risk of the disease from just below $1 \%$ in the general population to over $40 \%$ in monozygotic twin studies ${ }^{1}$, leading to extensive searches for the genetic basis of the disease. It was first proposed in the early

\section{(c) The Author(s) 2018}

(c) (i) Open Access This article is licensed under a Creative Commons Attribution 4.0 International License, which permits use, sharing, adaptation, distribution and reproduction c. in any medium or format, as long as you give appropriate credit to the original author(s) and the source, provide a link to the Creative Commons license, and indicate if changes were made. The images or other third party material in this article are included in the article's Creative Commons license, unless indicated otherwise in a credit line to the material. If material is not included in the article's Creative Commons license and your intended use is not permitted by statutory regulation or exceeds the permitted use, you will need to obtain permission directly from the copyright holder. To view a copy of this license, visit http://creativecommons.org/licenses/by/4.0/. 
1970s that inadequacy of tonically active inhibitory GABAergic neuronal activity relative to excitatory neuronal activity initiates the disease ${ }^{2}$, and evidence supports the etiological participation of GABAergic neurons including parvalbumin-containing ones in the hippocampus $^{3-5}$. At present, neural elements that are recognized to play important roles in schizophrenia include contribution of dopaminergic neurotransmission dysfunction to the genesis of psychotic symptoms and abnormalities of neuronal connectivity possibly involving interneurons ${ }^{6}$.

The first identification of a GABAergic pathway gene as a robust schizophrenia candidate gene was obtained by us based on the association of intronic single nucleotide polymorphisms (SNPs) in a 3551-bp segment in the vicinity of the AluYi6AH-151 insertion in the GABA $_{\mathrm{A}}$ receptor $\beta_{2}$ subunit gene (GABRB2) with schizophrenia in Chinese $^{7}$, and later confirmed in other populations ${ }^{8,9}$. Genotype-dependent expression and alternative splicing of the GABRB2 transcript gave rise to reduced gene expression as well as decreased long-to-short isoform ratio of the $\beta_{2}$ subunit in postmortem schizophrenic brains, and $\mathrm{GABA}_{\mathrm{A}}$ receptors carrying the long isoform were positively selected, and more quickly fatigued electrophysiologically than those carrying the short isoform upon repeated stimulation ${ }^{10-13}$. Gene expression was subjected to epigenetic regulations, including partial maternal imprinting, which were perturbed in schizophrenia $^{14,15}$. These findings established not only GABRB2 as a robust candidate gene, but also a chain of correlations leading from genotypes to alternative splicing and electrophysiological alteration. Moreover, the schizophreniaassociated genotypes of GABRB2 in the AluYi6AH-151 region were found to be associated with bipolar dis$\operatorname{order}^{16}$, heroin addiction ${ }^{17}$, and both positive symptoms in schizophrenia patients and altruism in normal subjects ${ }^{18}$.

More recently, genome-wide association studies (GWAS) identified 108 genetic loci related to schizophrenia that did not include $G A B R B 2^{19}$. On the other hand, when 40 polymorphisms in 12 'top' candidate genes for schizophrenia including $A K T 1$, COMT, $D A O, D R D 2$, DRD4, DTNBP1, GABRB2, IL1B, MTHFR, PPP3CC, SLC6A4, and TP53 with familial association data were subjected to meta-analysis, only the disease association of rs1816072 upstream of AluYi6AH-151 in GABRB2 remained significant after correction for multiple testing ${ }^{20}$. In view of this, the objective of the present study was to examine the relationship between the $\mathrm{GABA}_{\mathrm{A}} \beta_{2}$ subunit gene dosage and possible schizophrenia-like symptoms in an animal model. Previously, homozygous Gabrb2knockout mice were found to display normal body weight, elevated level of locomotor activity and loss of more than $50 \%$ of total $\mathrm{GABA}_{\mathrm{A}}$ receptors ${ }^{21}$. In addition, they exhibited reduced duration of loss of the righting reflex due to alcohol and sleep-time induced by $\mathrm{GABA}_{\mathrm{A}}$ receptor ligands ${ }^{22}$, outer hair cell dysfunction in the cochlea ${ }^{23}$, as well as a novel form of inhibitory synaptic plasticity in the cerebellum $^{24}$. In the present study, Gabrb2-knockout mice of both homozygous ( $\mathrm{KO}$ or Gabrb2 $2^{-1-}$ ) and heterozygous (HT or Gabrb2 $2^{+-}$) genotypes were compared to wild-type (WT or Gabrb2 $2^{+/+}$) mice regarding the possible presence of schizophrenia-like phenotypes. The results obtained provided evidence for a GABRB2-origin of schizophrenia.

\section{Materials and methods \\ Animals}

Gabrb2 HT transgenic mice (C57BL/6-129/SvEv hybrid) ${ }^{21}$ supplied by Taconic Farms, Inc. (New York) were bred to yield WT, HT, and KO mice that were weaned at week-3, genotyped using primers specific for the Gabrb2 and Neo genes (Supplementary Methods) and housed in groups of four to six with water and food ad lib, with a 12-h light cycle from 08:00 to 20:00. All animal experiments were pre-approved by the Animal Ethics Committee of HKUST and conducted in accordance with The Code of Practice for Care and Use of Animals for Experimental Purposes. The Code follows international guidelines of animal welfare and is approved by the Department of Health and the Fisheries and Conservation Department of HKSAR.

\section{Antibodies and immunofluorescent reagents}

ELISA kits for TNF- $\alpha$ and IL- 6 were obtained from Invitrogen, USA. Reagents for immunohistochemical analysis included primary antibodies against NeuN (1:500, monoclonal, clone A60; EMD Millipore, MA), GFAP (1:500, rabbit polyclonal; Boster Biological Technology, CA), DCX (1:200, goat polyclonal; Santa Cruz Biotechnology, TX), Iba1 (1:500, rabbit polyclonal; Wako, Japan), and parvalbumin (PV, 1:3000, rabbit polycolonal; Proteintech, IL). The fluorescence-labeled secondary antibodies FITC-conjugated donkey anti-mouse IgG for NeuN, Cy3-conjugated donkey anti-rabbit IgG for GFAP, Iba1, FITC-conjugated donkey anti-rabbit IgG for PV, and Cy3-conjugated donkey anti-goat IgG for DCX were obtained from Jackson ImmunoResearch Inc., PA.

\section{Behavioral tests}

Male mice, 8-10 weeks old were employed in the behavioral tests except for social behavior which employed 9-10-weeks-old female mice. Both genders were employed in the epilepsy tests, and 3-weeks-old mice were also used in the audiogenic epilepsy test. Behavioral tests were conducted based on previous protocols ${ }^{25-34}$ (Supplementary Methods). Fertility was monitored in 8week-old mice. To examine parent-of-origin effects, paternal HT (HT-P) and maternal HT (HT-M) mice were 
generated by mating WT male with $\mathrm{KO}$ female and $\mathrm{KO}$ male with WT female, respectively. Animals from multiple litters were grouped and tested randomly and the experimenters were blinded to the genotype of the mice.

\section{Biochemical analysis and immunoassays}

Tissues were collected from male mice aged 10-11 weeks old. Brain and liver tissues were homogenized in phosphate buffer saline (PBS) at $\mathrm{pH} 7.2$ and subjected to MDA analysis by reaction with thiobarbituric acid $(\mathrm{TBA})^{35}$. Blood sample was collected by cardiac excision from anesthetized mouse using a syringe prewashed with $0.5 \%$ heparin in saline. After centrifuging the blood sample at $1500 \times g$ for $10 \mathrm{~min}$ at $4{ }^{\circ} \mathrm{C}$, plasma was collected and subjected to reactive oxygen metabolite (ROM) analysis by reaction with $N, N$-dimethyl- $p$-phenylenediamine to quantitate tert-butylhydroperoxide (tBHP) equivalents ${ }^{36}$. ELISA kits for TNF- $\alpha$ and IL-6 (Invitrogen, USA) were used for brain assays according to manufacturer's instructions.

\section{Immunohistochemical analysis}

Male mice aged 10-11 weeks old were used. Cellular immunofluorescence was quantitated based on number of fluorescent cells or optical density using fluorescencelabeled antibodies ${ }^{37}$. Detailed methods are described in Supplementary Methods.

\section{Quantitative real-time PCR assays of mRNA expression}

The mRNA expressions of various $\mathrm{GABA}_{\mathrm{A}}$ receptor subunits in cerebrum and cerebellum tissues extracted from 10 to 11-weeks-old male mice were measured by real-time PCR (RT-PCR) using QuantiTect ${ }^{\circledR}$ reverse transcription kit (Qiagen) as described ${ }^{38}$. Briefly, total RNA was extracted from fresh brain tissue using Trizol reagent (Invitrogen Corp., CA) according to manufacturer's instruction. Total RNA was converted to cDNAs using QuantiTect, and RT-PCR was performed using FastStart Universal SYBR Green Master (Roche) on the 7500 Real-Time PCR System (Thermo Fisher Scientific). Actb and Pgk1 mRNAs were employed as reference. The primers employed in these RTPCR experiments are described in Supplementary Table S1.

\section{Statistical analysis}

Samples sizes were based on established practice and on our previous experience in respective assays ${ }^{26,34}$, and to some extent determined by breeding availability. The number of independent samples in each group is indicated by the individual points in the graphs, and also in the figure legends. Experimenters were blinded to animal genotype in the behavioral experiments and no sample was excluded from analysis. All data were presented as mean \pm SEM. Statistical analysis was performed using Prism 5.0 (Graph Pad Software, La Jolla, CA, USA) and statistical significance was set at $p<0.05$. Immunohistochemical data were analyzed by two-tailed unpaired $t$-test. All other data were analyzed using either one-way ANOVA followed by Newman-Keuls post-hoc test when one variable, i.e., genotype, was tested; or two-way ANOVA followed by Dunnett's posthoc test when two variables, i.e., genotype and drug dosage or genotype and gender, were tested. The statistical test used for each analysis is indicated in each figure legend and all data met the assumptions of the statistical tests. The variance between the statistically compared groups are similar.

\section{Results}

\section{Compromised fertility in naive knockout mice}

Naive KO mice displayed compromised fertility compared to WT mice, with $p<0.05$ (Supplementary Table S2). Average litters of $<4$ were obtained when both parents were naive $\mathrm{KO}$, as opposed to litters of more than 5 when naive male $\mathrm{KO}$ were mated with either naive female HT or WT. Normal litters of more than 6 were obtained for all other combinations of naive or non-naive parents.

\section{Reduced affective symptoms}

When the depression and anxiety levels of HT and KO mice were assessed, the $\mathrm{KO}$ mice displayed significantly reduced immobility time in the tail-suspension test as well as increased sucrose preference compared to WT mice; whereas the HT mice displayed a smaller reduction in tailsuspension time and no significant increase in sucrose preference (Fig. 1a, b). Thus KO, and to a lesser extent $\mathrm{HT}$, displayed a reduced level of depression relative to WT. In the elevated-plus maze test for anxiety, KO but not HT exhibited a significant increase of percentile entry into the open arms that was indicative of a decreased level of anxiety (Fig. 1c).

\section{Locomotor hyperactivity and behavioral stereotypy}

When psychotic agitation was assessed based on locomotor hyperactivity and behavioral stereotypy, both $\mathrm{KO}$ and HT mice displayed locomotor hyperactivity compared to WT, with KO being more hyperactive than HT, showing that Gabrb2 gene dosage was negatively correlated with locomotor activity (Fig. 2a). Regarding behavioral stereotypy, more rearing and climbing were observed in KO compared to both WT and HT, whereas there was no significant difference between WT and HT, again demonstrating the presence of gene dosage effects (Fig. 2b). There was no significant difference between KO, HT, and WT in either circling or sniffing stereotypy (Supplementary Fig. S1a, b). 


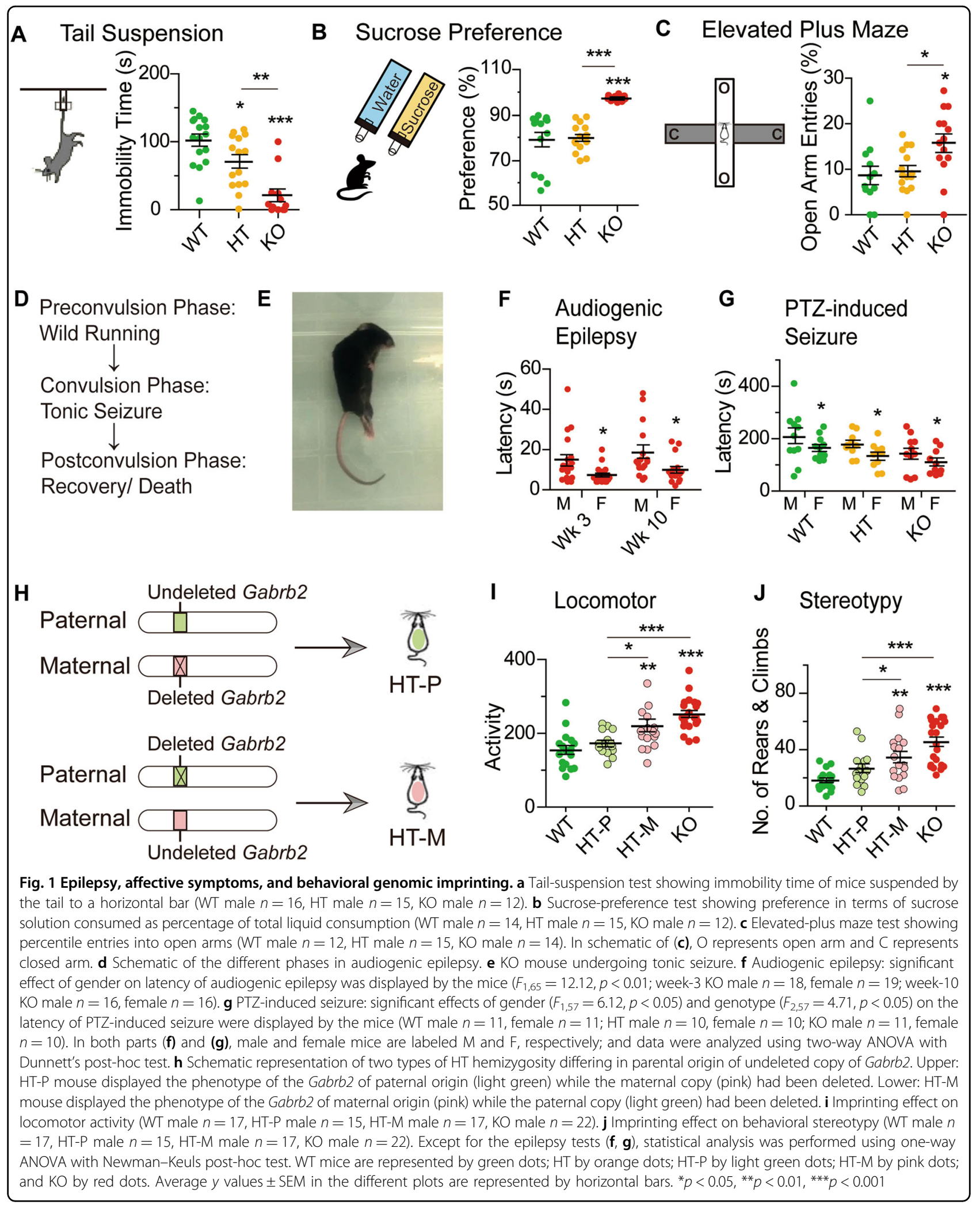




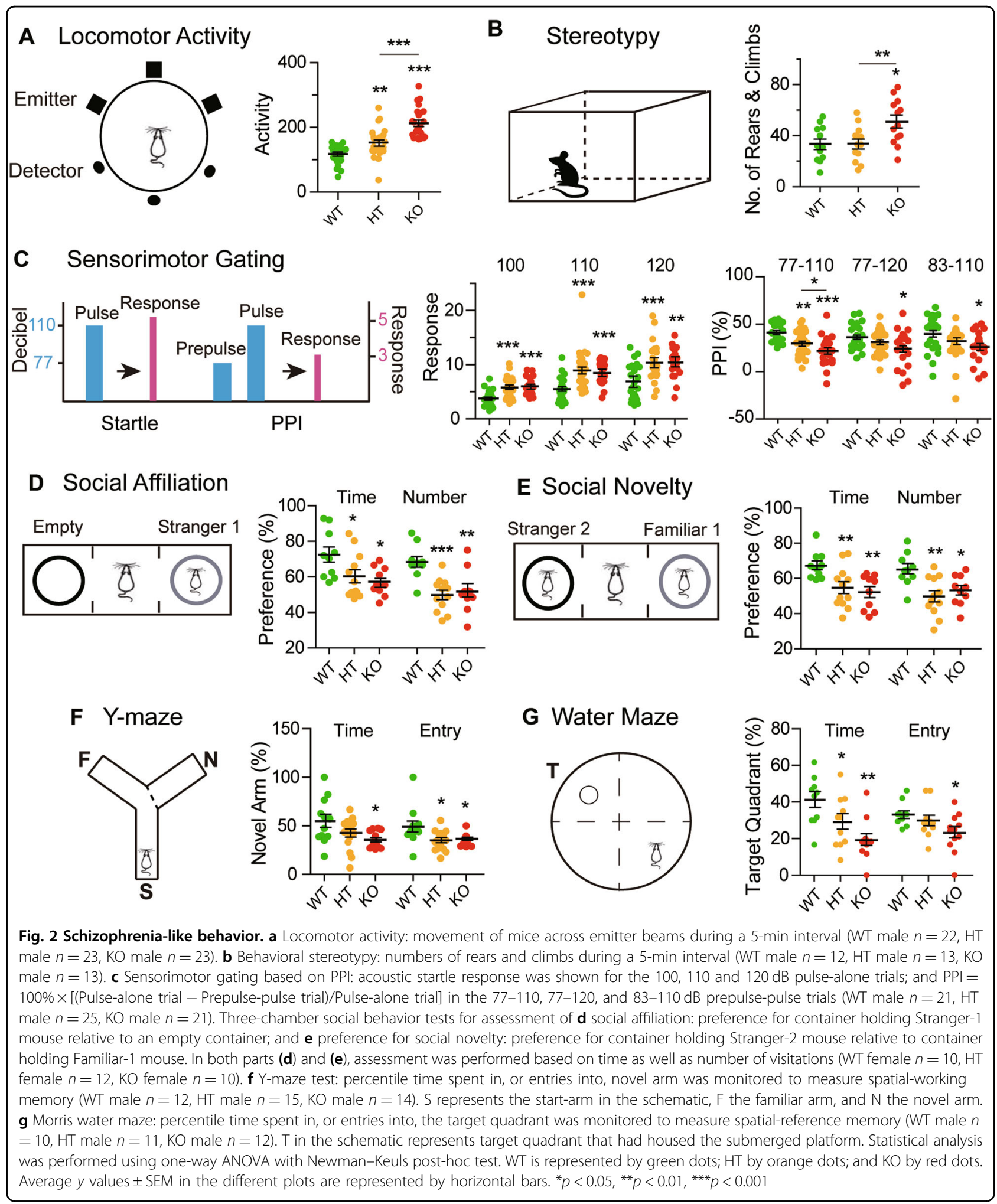

\section{Sensorimotor gating deficit}

In the PPI test for core schizophrenia-like symptoms relating to sensorimotor gating, the acoustic startle response was significantly larger in $\mathrm{HT}$ and $\mathrm{KO}$ mice than in WT mice for 100,110 , and $120 \mathrm{~dB}$ sounds, suggesting that both $\mathrm{HT}$ and $\mathrm{KO}$ were more sensitive to acoustic stimulus than WT. Significant decreases in PPI were observed in KO relative to WT for the 77-110, 77-120, 
and $83-110 \mathrm{~dB}$ prepulse-pulse combinations, in HT for only the $77-110 \mathrm{~dB}$ combination (Fig. 2c), and in neither KO nor HT for the $83-120 \mathrm{~dB}$ combination (Supplementary Fig. S1c), indicating that reduced copies of Gabrb2 brought about sensorimotor gating deficits resembling those observed in schizophrenia.

\section{Deficits in social functions}

In the three-chamber test, WT displayed greater social affiliation and preference for social novelty compared to $\mathrm{HT}$ and $\mathrm{KO}$ based on either time or number of close contact visitations, thereby establishing deficits of $\mathrm{HT}$ and $\mathrm{KO}$ with respect to both social affiliation and preference for social novelty (Fig. 2d, e). Since there was no significant difference between $\mathrm{HT}$ and $\mathrm{KO}$ regarding either time or number of visitations when they were compared against one another, no gene dosage effect was evident.

\section{Impairment of cognitive functions}

For hippocampus-dependent memory functions, the Ymaze measures spatial-working memory, and the Morris water maze measures spatial-reference memory ${ }^{39}$. In the Y-maze test, reduced novel-arm visitation time and entries were displayed by $\mathrm{KO}$ mice, but only reduced entries were displayed by HT mice (Fig. 2f). In the Morris water maze test, both reduced target-quadrant visitation time and entries were displayed by $\mathrm{KO}$ mice, but only reduced visitation time was displayed by HT mice (Fig. $2 \mathrm{~g})$. Therefore, the KO and HT mice were inflicted with deficits in both spatial-working memory and spatialreference memory, with significant gene dosage effect.

\section{Audiogenic epilepsy and chemical-induced seizure with gender effect}

When exposed to white noise, $95 \%$ or more of both female and male KO mice, but none of the HT and WT mice, were susceptible to audiogenic epilepsy characterized by wild running followed by tonic seizure (Fig. 1d-f, Supplementary Video, Supplementary Table S3); whereas PTZ induced seizure in WT, HT as well as KO mice (Fig. $1 \mathrm{~g})$. For audiogenic seizure, the average latency to seizure was shorter for female $\mathrm{KO}$ than for male $\mathrm{KO}$ at both week- 3 and week-10; and both $\mathrm{KO}$ and HT were more susceptible than WT toward PTZ-induced seizure with the females again displaying a shorter latency than the males in each of the WT, HT, and KO groups (Fig. 1f, g). The susceptibility of $\mathrm{KO}$ mice to audiogenic epilepsy was in accord with the findings of comorbidity between schizophrenia and epilepsy ${ }^{40,41}$.

\section{Parent-of-origin effects}

Parent-of-origin effects were examined by dividing HT mice into the paternal HT (HT-P) and maternal HT (HT$\mathrm{M})$ groups, with acquisition of a normal copy of Gabrb2 from father or mother respectively (Fig. 1h). HT-M mice exhibited lower levels of locomotor hyperactivity and stereotypy of repetitive climbing and rearing than $\mathrm{KO}$ mice but higher than both HT-P and WT mice in theses regards, whereas there was no significant difference between HT-P and WT mice (Fig. 1i, j). On the other hand, with respect to the level of depression assessed by the tail-suspension test, both HT-P and HT-M exhibited significantly shorter immobility time than WT and longer immobility time than $\mathrm{KO}$, with no significant difference between HT-P and HT-M (Supplementary Fig. S1d).

\section{Reversal of phenotypic alterations by risperidone and diazepam}

The antipsychotic drug risperidone has been employed for the clinical treatment of schizophrenia ${ }^{42}$. Administration of $0.3 \mathrm{mg} / \mathrm{kg}$ risperidone i.p. significantly reversed the PPI inhibition to levels similar to or even higher than those of untreated WT (Fig. 3a-c). Locomotor activity was suppressed in WT, HT and KO; the suppression in $\mathrm{HT}$, but not that in $\mathrm{KO}$, sufficed to bring the activity level down to that of untreated WT (Fig. 3d). The increased numbers of stereotypic rears and climbs in both HT and $\mathrm{KO}$, however, could be rolled back completely (Fig. 3e). There was a small reversal of the cognitive defect of $\mathrm{KO}$ by the drug in the Morris water maze (Fig. 3f), but no significant effect on the Y-maze test or social behavior tests (Supplementary Fig. S2). The drug prolonged the immobility time in the WT, HT, and KO mice in the tailsuspension test (Fig. 3g), and reduced the percentage time spent by $\mathrm{HT}$ and $\mathrm{KO}$ mice in the open arms on the elevated-plus maze (Fig. 3h), indicating its effectiveness in partially reversing the decreased level of depression, and fully reversing the decreased level of anxiety in HT and KO mice.

Upon administration of 0.3 or $0.5 \mathrm{mg} / \mathrm{kg}$ i.p. of the $\mathrm{GABA}_{\mathrm{A}}$ receptor agonist diazepam to the $\mathrm{KO}$ mice, the latency to audiogenic epilepsy was increased in both male and female $\mathrm{KO}$ mice (Fig. 3i), and the prevalence of audiogenic epilepsy was reduced (Supplementary Table S3). However, $0.5 \mathrm{mg} / \mathrm{kg}$ diazepam i.p. also induced sedation based on the holeboard test (Supplementary Fig. S3).

\section{Immunohistochemical and biochemical alterations in the brain}

There was no extensive change in the optical density (O. D.) of neuronal immunostaining obtained with fluorescent-labeled anti-NeuN between $\mathrm{KO}$ and WT mice in the anterior cingulate cortex (ACC) or hippocampus $(\mathrm{HC})$, or the number of PV-positive neurons in the dentate gyrus (DG) in hippocampus (Fig. 4a-c, g). In contrast, the O.D. of PV-staining in neurons in the ACC, and the number of PV-positive neurons in the piriform cortex 


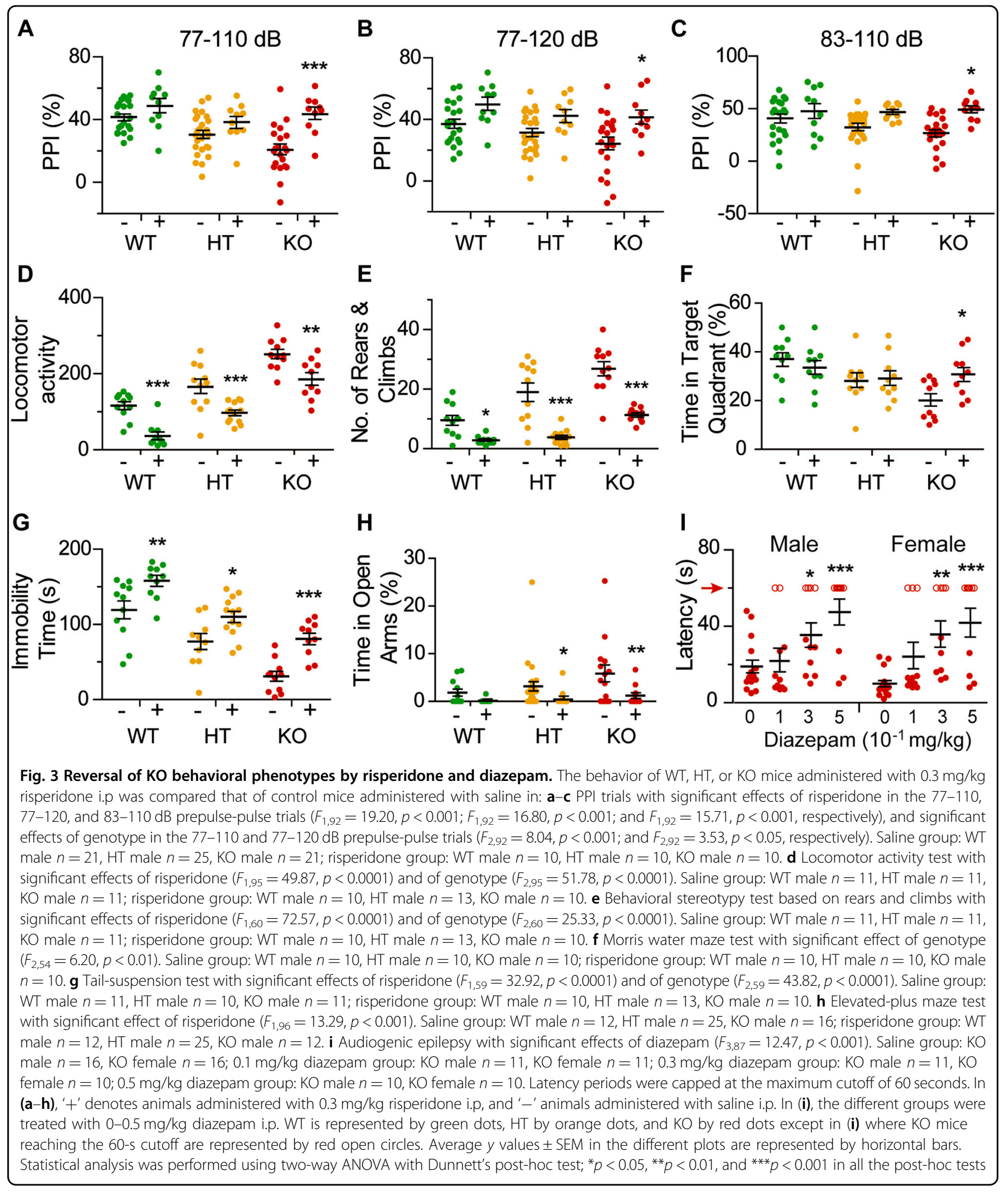

(PC) were decreased in $\mathrm{KO}$ mice relative to WT mice (Fig. $4 \mathrm{e}, \mathrm{f})$. The number of DCX-positive newborn neurons in DG, and the O.D. of GFAP-positive astrocytes in CA1 and DG of hippocampus were all decreased in $\mathrm{KO}$ compared to WT (Fig. 4d, h, i). There was no significant change in the O.D. of GFAP-positive astrocytes in ACC, or the number of PV-positive neurons in CA1 in hippocampus or central amygdaloid nucleus (CEA) in KO compared to 


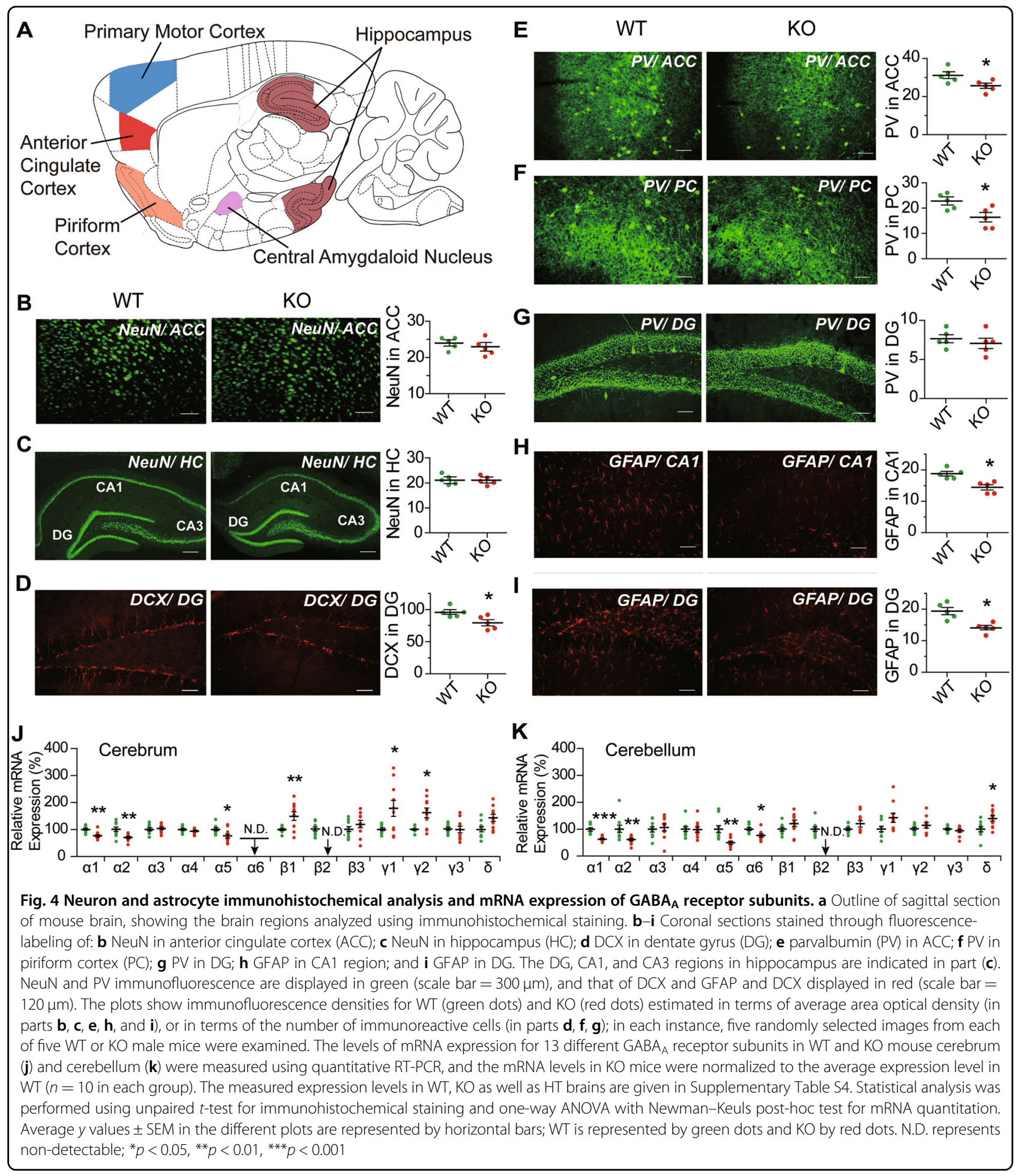

WT (Supplementary Fig. S4). On the other hand, the number of Iba1-positive microglia was increased in $\mathrm{KO}$ compared to WT mice in CA1, DG, ACC, CEA, and PC, but not in the primary motor cortex (PMC) (Fig. 5a-f).

The brain levels of oxidative stress measured by MDA were elevated in HT and KO compared to WT mice, with significant dosage effect between $\mathrm{HT}$ and $\mathrm{KO}$ (Fig. 5g), but neither in liver nor blood as measured by ROM (Supplementary Fig. S5). The brain levels of the inflammatory cytokines TNF- $\alpha$ and IL- 6 were elevated in KO, but not in HT, compared to WT mice (Fig. 5h, i). 


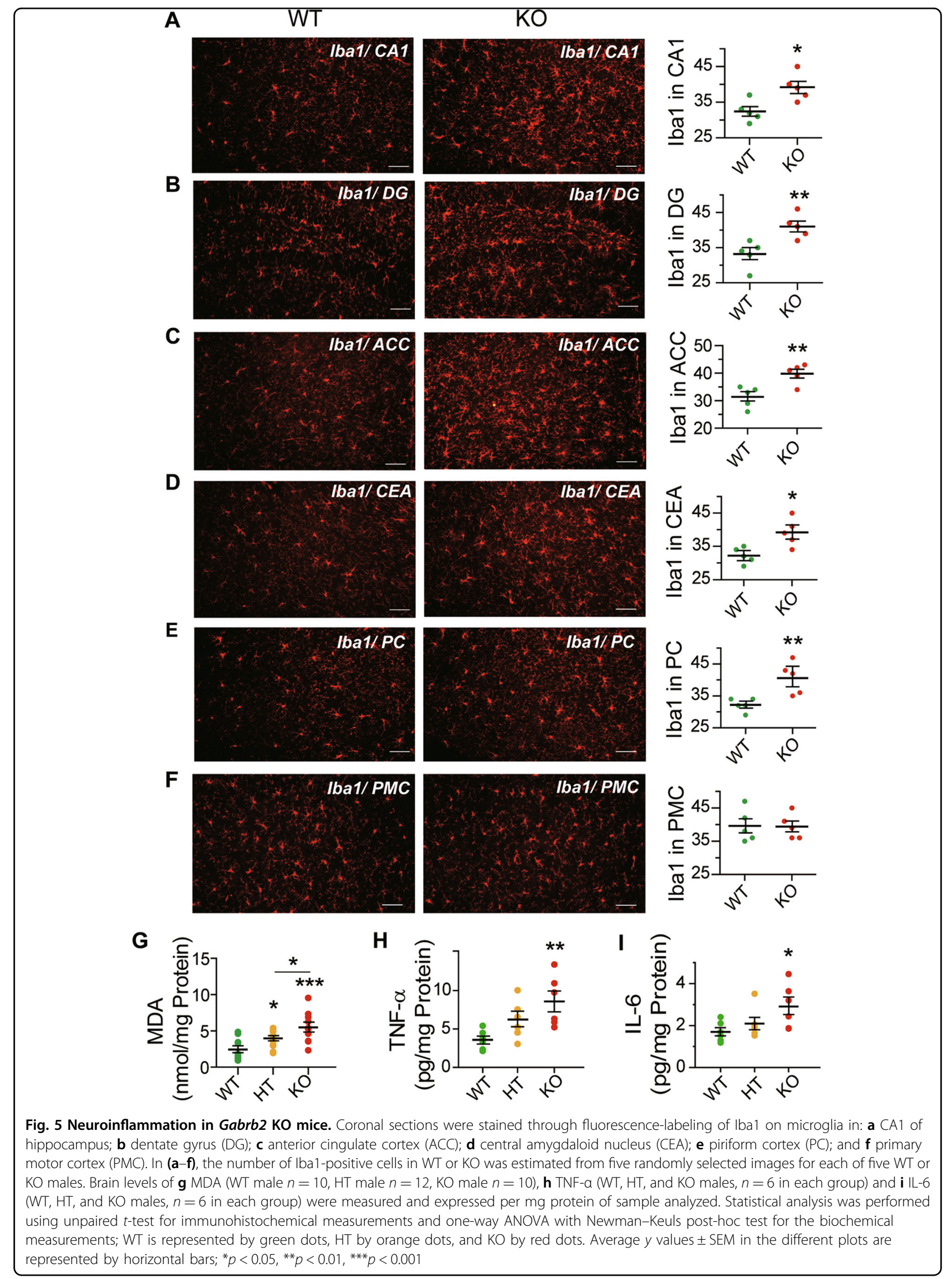




\section{Differential expression of $\mathrm{GABA}_{\mathrm{A}}$ receptor subunits}

When Gabrb2 exon 7-specific primers were employed to quantitate Gabrb2 expression based on its mRNA level, expression was found to be reduced in HT mice compared to WT, and non-detectable (N.D.) in KO, in both cerebrum and cerebellum. The mRNA levels of different subunits of $\mathrm{GABA}_{\mathrm{A}}$ receptors in the cerebrum or cerebellum in KO (red dots) compared to WT (green dots) showed significant upregulations for Gabrb1, Gabrg1, and Gabrg2, but downregulations for Gabra1, Gabra2, and Gabra5 in the KO cerebrum; in contrast, there were significant upregulation for Gabrd, but downregulations for Gabra1, Gabra2, Gabra5 and Gabra6 in the KO cerebellum (Fig. 5j, k, Supplementary Table S3). A factor underlying the various downregulations was likely the loss of more than $50 \%$ of total $\mathrm{GABA}_{\mathrm{A}}$ receptors in the $\mathrm{KO}$ mice $^{21}$, and the various upregulations could be indicative of replacement of the missing $\beta_{2}$ subunit in $\mathrm{GABA}_{\mathrm{A}}$ receptors by the upregulated subunits.

\section{Discussion}

Previously, analysis of schizophrenia genomics pointed to $G A B R B 2$ as a key susceptibility gene for schizophrenia $^{7-13}$. Alternative splicing of the gene transcript gave rise to two major products comprising an ancient short $\beta_{2}$ isoform and a derived, positively selected long $\beta_{2}$ isoform, and only the long isoform carries a potential phosphorylation site at $\mathrm{Thr}^{365}$. $\mathrm{GABA}_{\mathrm{A}}$ receptors bearing the long $\beta_{2}$ isoform were fatigued and underwent current rundown more rapidly that those with the short isoform upon repeated stimulation in the presence of low ATP concentration, thereby diminishing the strength of inhibitory signaling of GABAergic neurons. This effective linkage of neuroinhibition to energy supply made possible by the long isoform was positively selected in evolution, for it would disinhibit actions related to striving, hunting, and pursuit when food is limited. There were reductions of $21.7 \%$ in long-isoform expression, $13.4 \%$ in short isoform expression and $15.8 \%$ in their combined expression in schizophrenic brain, resulting in twin deficits of total $\beta_{2}$ subunit and the long isoform relative to short isoform ${ }^{12}$.

\section{Pathophysiological mechanisms of Gabrb2 knockout}

In agreement with schizophrenia genomics, Figs. 1 to 5 show that the Gabrb2 KO mice exhibited behavioral and cognitive changes similar to those observed in schizophrenia, including: neuroinflammation with increased oxidative stress $^{43}$, increased pro-inflammatory cytokines $^{44}$, and microglial activation ${ }^{45-47}$, as well as the comorbidities of hyperactivity ${ }^{48}$, decreases in cell proliferation in hippocampus ${ }^{4,49,50}$, and epilepsy ${ }^{40,41}$.

Based on immunostaining, the complete loss of Gabrb2 affected four types of cells in the brain prominently: viz. decreases in DCX-positive newborn neurons, PV-positive neurons and GFAP-positive astrocytes, together with widespread increases in Iba1-positive microglia (Fig. 4b-i, Fig. 5a-e). Since PV-positive GABAergic interneurons are important to gamma-band synchrony and cognition ${ }^{51,52}$, their decreases could contribute to the deficits in cognition revealed by the Y-maze and water maze tests (Fig. 2f, g). The increases in Iba1-positive microglia together with increased brain levels of oxidative stress and the proinflammatory cytokines TNF- $\alpha$ and IL- 6 (Fig. $5 \mathrm{~g}-\mathrm{i}$ ) pointed to the presence of regional neuroinflammation. Notably, the decreases of newborn neurons and astrocytes along with microglia activation in the hippocampus underlined the importance of hippocampus in the phenotypes of Gabrb2knockout mice (Fig. 2f, g), and confirmed earlier suggestions of hippocampal involvement in schizophrenia ${ }^{4,5}$.

Unlike classical phasic $\mathrm{GABA}_{\mathrm{A}}$ receptor-mediated inhibition, tonic $\mathrm{GABA}_{\mathrm{A}}$ receptor-mediated inhibition results from the activation of extrasynaptic receptors by low concentrations of ambient GABA in the extracellular space ${ }^{53}$, and tripartite synapses formed by presynaptic neuron, postsynaptic neuron and astrocyte enable bidirectional communication between astrocytes and neurons ${ }^{54}$. Moreover, the GABAergic astrocytes modulate the GABAreceptive microglia, and GABA suppresses the reactive response of both astrocytes and microglia to inflammatory stimulants, leading to a reduced release of the inflammatory cytokines IL- 6 and TNF- $\alpha^{55,56}$. Evidence also suggests the participation of neuron-astrocyte-microglia triad in the regulation of neuroinflammation in hippocampus ${ }^{57}$. Accordingly, the observed decreases in newborn neurons, PV-positive interneurons and astrocytes, and increases in microglia (Figs. 4 and 5) may be expected to alter significantly the interactions between interneurons, astrocytes and microglia, giving rise to regional neuroinflammation and development of schizophrenia-like and comorbid phenotypes in the Gabrb2-knockout mice.

A substantial portion of schizophrenia cases experience episodes of mood disorder as well as periods of nonaffective psychosis, and the distinction between schizophrenia and schizoaffective disorder is marked with some difficulty $^{58}$. The decreased depression-like behavior in the tail-suspension and sucrose-preference tests, and anxietylike behavior in the elevated-plus maze in Gabrb2knockout mice (Fig. 1a-c), indicated a separation of the schizophrenia type symptoms from comorbid affective symptoms. Insofar that anxiety could be regulated through extrasynaptic inhibition ${ }^{59}$, the alterations in GABAergic interneurons and tripartite synapses brought about by Gabrb2 knockout could also be factors in the decreases of depression- and anxiety-like symptoms.

\section{Comparison of animal models for schizophrenia}

A variety of animal models have produced limited phenotypic resemblances with schizophrenia and its 
comorbidities, as in the case of Disc-1, Nrg1, and ErbB4 knockouts; and in some instances included also phenotypic divergences, as in the case of unimpaired hippocampal-dependent memory and lack of socialinteraction reduction in amphetamine models, lack of sustained PPI deficit in phencyclidine models, increased PPI in dysbindin knockout, and reduced locomotion in reelin knockout ${ }^{60}$. Likewise, there were lack of socialinteraction deficit in dopamine-transporter knockout ${ }^{61}$; decreased locomotor activity, and lack of significant change in PPI or learning and memory in Gabra1 knockout $^{62}$; and improved performance in the water maze test in Gabra5 knockout ${ }^{63}$. Mice hypomorphic in the $N$ methyl-D-aspartate (NMDA) R1 subunit (NR1) exhibited decreased PPI and social affiliation ${ }^{64}$, but the induction of schizophrenia-like symptoms by partial ablation of NR1 from GABAergic neurons, a majority of which were parvalbumin-positive, pointed to the mediation of schizophrenia-like phenotypes by NMDA-receptors on GABAergic neurons ${ }^{65}$, in which case the schizophrenialike phenotypes could stem from disturbance of either the NMDA-receptors or $\mathrm{GABA}_{\mathrm{A}}$-transmission or both. In contrast, in Gabrb2 knockout, the absence of the $\mathrm{GABA}_{\mathrm{A}^{-}}$ receptor $\beta_{2}$ subunit was clearly the root of the schizophrenia-like phenotypes and comorbidities of the $\mathrm{KO}$ mice, with no major divergence from the positive symptoms, negative symptoms and cognitive deficit of schizophrenia, and reversible in part or in full by risperidone for a number of the symptoms. Furthermore, the dissimilarities between the symptoms of schizophrenia and those observed so far in the Gabra1 and Gabra5 knockouts suggest that the extensive similarities between Gabrb2 knockout and schizophrenia are not readily shared by knockouts of other subunits of $\mathrm{GABA}_{\mathrm{A}}$ receptors.

\section{GABRB2-origin of schizophrenia}

Earlier findings on schizophrenia genomics, gene expression and alternate-splicing of the $\beta_{2}$ receptor subunit have pointed to a key role played by GABRB2 genotypes and haplotypes in the disease. However, because schizophrenia and its comorbidities are associated with such a variety of symptoms, it is difficult to determine the minimum number of genetically perturbed genes required for disease initiation. In this regard, the Gabrb2-knockout model demonstrated that deletion of Gabrb2 alone was sufficient cause for a range of the schizophrenia-like positive symptoms, negative symptoms and cognitive impairments in the homozygous $\mathrm{KO}$ mice, thereby enabling the proposal of a GABRB2-origin theory of schizophrenia. That the hemizygous HT mice were symptomatic indicated clearly just how low is the inhibitory-power redundancy in some of the $\beta_{2}$-containing GABAergic receptors in the brain. The twin reductions in total $\beta_{2}$ expression and long-to-short $\beta_{2}$ isoform ratio induced by the schizophrenia susceptibilityenhancing genotypes and haplotypes in the AluYi6AH151 region of GABRB2 would thus lead to inadequate inhibitory power on account of $\beta_{2}$ shortage and diminished long-to-short isoform ratio, and therefore illness.

To trace the etiological pathway from a shortage of $\beta_{2}$ subunit, especially its long isoform, it may be noted that neural stability depends on a balance between excitatory neurotransmitters such as glutamate, and inhibitory ones comprising mainly GABA. In the face of a shortage of any $\mathrm{GABA}_{\mathrm{A}}$ subunit, risk of disinhibition would be enhanced, particularly in brain regions such as hippocampus where the neurons are $\sim 90 \%$ glutamatergic pyramidal cells, and only $10 \%$ GABAergic non-pyramidal cells ${ }^{66}$. The nonlethality of two-copy Gabrb2 knockout in mice ${ }^{21}$ show that the brain can extensively maintain function by replacing the $\beta_{2}$ subunit in $\mathrm{GABA}_{\mathrm{A}}$ receptors with other subunits, and the subunit upregulations in $\mathrm{KO}$ mice suggest that, upon deletion of Gabrb2, the $\beta_{2}$ subunit in $\mathrm{GABA}_{\mathrm{A}}$ receptors could be replaced by $\beta_{1}, \gamma_{1}$, and $\gamma_{2}$ in the cerebrum, or by $\delta$ in the cerebellum (Fig. 4j, k). Since there are $\beta_{1}$ and $\gamma_{2}$ but limited $\delta$ and little $\gamma_{1}$ in the hippocampus available for $\beta_{2}$ replacement ${ }^{67}$, insufficient or unsatisfactory replacement of $\beta_{2}$ by other subunits in some hippocampal $\mathrm{GABA}_{\mathrm{A}}$ receptors in the event of a $\beta_{2}$ deficit could represent a significant factor of defective hippocampal function in schizophrenia or in the Gabrb2 KO mice.

With disinhibition, overstimulation of neurons by glutamate leads to influx of calcium, excitotoxicity, and cell death $^{68}$, producing cell debris. Microglia activation is inducible by pathogens or cell debris, and the occurrence of microglial activation in various brain regions but not the primary motor cortex (Fig. 5a-f) indicated that microglial activation in the $\mathrm{KO}$ mouse brain was regional and induced by cell debris rather than infection. Microglial activation and neuroinflammation could in turn give rise to the symptoms and comorbidities of schizophrenia $^{45-47}$. The immune-response nature of microglial activation is consistent with the association of schizophrenia with the major histocompatibility complex $(\mathrm{MHC})^{19,69}$. In severe traumatic brain injury, excitotoxicity gives rise to posttraumatic epilepsy in $20 \%$ of cases, and $50 \%$ in cases with penetrating head wounds ${ }^{70}$, indicating that the audiogenic epilepsy of the Gabrb2 KO mice could likewise be the consequence of excitotoxicity. The observation of audiogenic epilepsy in only KO but not HT mice suggests that a deeper GABAergic deficit than that inflicted by Gabrb2 hemizygosity would be required for epileptogenesis.

In conclusion, the advantage of the GABRB2-origin theory of schizophrenia resides in the multiplicity of its supportive evidence: (a) The SNPs in the vicinity of the 
AluYi6AH-151 insertion of GABRB2 were correlated with schizophrenia with odds ratios of 1.93-2.50, and also with both total $\beta_{2}$ subunit and its long-to-short isoform ratio, indicating that their associations with schizophrenia arose directly from their regulation of $\beta_{2}$ expression and alternative splicing ${ }^{7-15}$. The evidence for their positive selection indicates their functional importance, haplotype analysis points to haplotypes $\mathrm{H} 26$ and $\mathrm{H} 73$ as protective, and $\mathrm{H} 19$ and $\mathrm{H} 81$ as risk-conferring toward schizophrenia $^{11}$, and the correlations between genotypes and antipsychotics dosage among schizophrenia patients ${ }^{16,18}$ confirm the clinical relevance of these SNPs. (b) The more rapid attenuation of long $\beta_{2}$ isoform-containing $\mathrm{GABA}_{\mathrm{A}}$ receptors compared to short isoform-containing ones in the presence of low ATP indicates that the long-to-short isoform ratio correlated to schizophrenia pertains to a clearly defined electrophyiological characteristic of $\beta_{2-}$ containing $\mathrm{GABA}_{\mathrm{A}}$ receptors. (c) Gabrb2 knockout in mice produced a range of schizophrenia-like symptoms and comorbidities, some of which are reversed partly or fully by the antipsychotic risperidone. (d) The theory can account for regional microglial activation in the brain of $\mathrm{KO}$ mice based on the varied $\mathrm{GABA}_{\mathrm{A}}$ receptor subunit compositions in different brain regions leading to unsatisfactory replacement of $\beta_{2}$ by other subunits in regions such as hippocampus and anterior cingulate cortex but not the primary motor cortex. (e) The theory can account for the otherwise difficult to explain complete recovery achieved in $25 \%$ of schizophrenia cases ${ }^{71}$ based on the $\sim 35 \%$ increases in total $\beta_{2}$-subunit expression and longto-short $\beta_{2}$ isoform ratio in human brains between the ages of 30 and 50, which would ameliorate by middle age the disease-eliciting shortages of $\beta_{2}$ subunit and its long isoform in adolescents and young adults ${ }^{15}$; and the marked differences in the compositions and presumably properties of $\mathrm{GABA}_{\mathrm{A}}$ receptors in early postnatal compared to adult rat brain ${ }^{72}$ may furnish a possible basis for the much lower incidence of schizophrenia in young children compared to adolescents. (f) The audiogenic epilepsy of KO mice helps to explain the comorbidity of epilepsy with schizophrenia. (g) The pervasive connections of disease-prone $\beta_{2}$-containing GABAergic interneurons in the brain could facilitate coalescence of the relatively minor defects of a large array of genes into a seriously debilitating multigene disease, thereby explaining the remarkably wide spectrum of schizophrenia phenotypes and comorbidities, as well as the finding of numerous schizophrenia SNPs with low odds ratios typically around 1.10 and rarely exceeding 1.20 , denoting only a very small effect on disease risk $^{73}$.

Based on the GABRB2-origin of schizophrenia, functionally defective $\beta_{2}$ subunit-containing $\mathrm{GABA}_{\mathrm{A}}$ receptors would begin the etiological changes, and proceed to involve wide ranging neuroreceptor systems and brain structures to produce the spectrum of symptoms and comorbidities characteristic of schizophrenia. This is evident from the possible modulation of PPI, which has been regarded as an endophenotype of schizophrenia, by interventions at the dopamine, NMDA, $5 \mathrm{HT}_{2 \mathrm{C}}, \mathrm{CB} 1$ cannabinoid, neurotensin-1, adenosine $\mathrm{A}(2 \mathrm{~A})$, alpha-7 nicotinic and histamine $\mathrm{H} 3$ receptors, revealing the participation of these diverse receptor systems in shaping a single disease phenotype ${ }^{74}$. On account of such networking between receptor systems, a GABRB2-origin of schizophrenia is entirely compatible with drug development against the disease targeting widely at the dopamine, glutamate, GABA, acetylcholine, serotonin, and hormonal systems, necessitated particularly by the shortfall of effective medications for the negative symptoms and cognitive deficits ${ }^{75,76}$. As illustrated in the present study, risperidone could moderate a number of the Gabrb2knockout-induced phenotypes even though the drug is known to act on catecholamine receptors (mainly dopamine 2, and alpha $1 \& 2$ adrenoceptors) and 5HT2 receptors ${ }^{42}$. Consequently, a deeper understanding of how different neurotransmitter and neuronal systems interact in schizophrenia to generate its symptoms and comorbidities, obtained through integrated approaches including clinical and genetic studies, investigation of postmortem schizophrenic brains and thorough analysis of the Gabrb2knockout model, will be important for not only delineation of the complex etiology of schizophrenia, but also expedited searches for improved drugs to treat the disease.

\section{Acknowledgements}

The study was supported by grants to HX from University Grants Committee of Hong Kong (No. DG13SC03), Ministry of Science and Technology of China

(National Science and Technology Major Project, No. 2017ZX09301064), and Shandong Province First Class Discipline Development Grant; National Natural Science Foundation of China Grant (No. 81471260) to Z.-H.X.; and HKJEBN Scholarship for Health and Quality Living to RKY. We are indebted to the Animal and Plant Care Facility at Hong Kong University of Science and Technology and the Laboratory Animal Unit at University of Hong Kong for use of facilities and assistance, Profs. H. Jiang and Z. Wang for supply of risperidone, and C.P. Lo and K.P. To for expert assistance. We thank Merck Sharp \& Dohme Corp. for provision of the Gabrb2-knockout mice.

\section{Author details}

'Division of Life Science, Applied Genomics Center and State Key Laboratory of Molecular Neuroscience, Hong Kong University of Science and Technology, Clear Water Bay, Hong Kong, People's Republic of China. ${ }^{2}$ Department of Neurobiology, Second Military Medical University, Shanghai, People's Republic of China. ${ }^{3}$ Department of Psychiatry, Li Ka Shing Faculty of Medicine, University of Hong Kong, Hong Kong, People's Republic of China. ${ }^{4}$ Shandong University of Traditional Chinese Medicine, Shandong, People's Republic of China

Conflict of interest

The authors declare that they have no conflict of interest.

\section{Publisher's note}

Springer Nature remains neutral with regard to jurisdictional claims in published maps and institutional affiliations. 
Supplementary Information accompanies this paper at (https://doi.org/ 10.1038/s41398-018-0176-9).

Received: 16 January 2018 Revised: 30 April 2018 Accepted: 4 June 2018 Published online: 17 July 2018

\section{References}

1. Sullivan, P. F., Kendler, K. S. \& Neale, M. C. Schizophrenia as a complex trait: evidence from a meta-analysis of twin studies. Arch. Gen. Psychiatry 60, 1187-1192 (2003)

2. Roberts, E. Prospects for research on schizophrenia. A hypothesis suggesting that there is a defect in the GABA system in schizophrenia. Neurosci. Res. Program Bull. 10, 468-482 (1972).

3. Perry, T. L., Kish, S. J., Buchanan, J. \& Hansen, S. Gamma-aminobutyricacid deficiency in brain of schizophrenic patients. Lancet 313, 237-239 (1979).

4. Benes, F. M. \& Berretta, S. GABAergic interneurons: implications for understanding schizophrenia and bipolar disorder. Neuropsychopharmacology 25, 1-27 (2001)

5. Lewis, D. A., Hashimoto, T. \& Volk, D. W. Cortical inhibitory neurons and schizophrenia. Nat. Rev. Neurosci. 6, 312-324 (2005).

6. Owen, M. J., Sawa, A. \& Mortensen, P. B. Schizophrenia. Lancet 388, 86-97 (2016).

7. Lo, W. S. et al. Association of SNPs and haplotypes in $\mathrm{GABA}_{\mathrm{A}}$ receptor beta2 gene with schizophrenia. Mol. Psychiatry 9, 603-608 (2004).

8. Lo, W. S. et al. GABRB2 association with schizophrenia: commonalities and differences between ethnic groups and clinical subtypes. Biol. Psychiatry 61, 653-660 (2007).

9. Petryshen, T. L. et al. Genetic investigation of chromosome $5 q$ GABA receptor subunit genes in schizophrenia. Mol. Psychiatry 10, 1074-1088 (2005).

10. Lo, W. S. et al. Positive selection within the schizophrenia-associated GABA(A) receptor beta(2) gene. PLoS ONE 2, e462 (2007)

11. $\mathrm{Ng}, \mathrm{S}$. K. et al. A recombination hotspot in a schizophrenia-associated region of GABRB2. PLOS ONE 5, e9547 (2010).

12. Zhao, $C$. et al. Two isoforms of $G A B A_{A}$ receptor $\beta_{2}$ subunit with different electrophysiological properties: differential expression and genotypical correlations in schizophrenia. Mol. Psychiatry 11, 1092-1105 (2006).

13. Zhao, $C$. et al. Alternative-splicing in the exon-10 region of $G A B A_{A}$ receptor $\beta_{2}$ subunit gene: relationships between novel isoforms and psychotic disorders. PLOS ONE 4, e6799 (2009).

14. Pun, F. W. et al. Imprinting in the schizophrenia candidate gene GABRB2 encoding GABA(A) receptor $\beta(2)$ subunit. Mol. Psychiatry 16, 557-568 (2011).

15. Zhao, C. et al. Epigenetic regulation on GABRB2 isoforms expression: developmental variations and disruptions in psychotic disorders. Schizophr. Res. 134 260-266 (2012).

16. Chen, J. et al. GABRB2 in schizophrenia and bipolar disorder: disease association, gene expression and clinical correlations. Biochem. Soc. Trans. 37, 1415-1418 (2009).

17. Kim, Y. S. et al. GABRB2 haplotype association with heroin dependence in Chinese population. PLOS ONE 10, e0142049 (2015).

18. Tsang, S. Y. et al. Social cognitive role of schizophrenia candidate gene GABRB2. PLOS ONE 8, e62322 (2013).

19. Schizophrenia Working Group of the Psychiatric Genomics Consortium. Biological insights from 108 schizophrenia-associated genetic loci. Nature 511, 421-427 (2014).

20. Shi, J., Gershon, E. S. \& Liu, C. Genetic associations with schizophrenia: metaanalyses of 12 candidate genes. Schizophr. Res. 104, 96-107 (2008).

21. Sur, $C$. et al. Loss of the major GABA(A) receptor subtype in the brain is not lethal in mice. J. Neurosci. 21, 3409-3418 (2001).

22. Blednov, Y. A. et al. Deletion of the alpha1 and beta2 subunit of GABAA receptors reduces actions of alcohol and other drugs. J. Pharmacol. Exp. Ther. 304, 30-36 (2003).

23. Maison, S. F., Rosahl, T. W., Homanics, G. E. \& Liberman, M. C. Functional role of GABAergic innervation of the cochlea: phenotypic analysis of mice lacking GABA(A) receptor subunits alpha 1, alpha 2, alpha 5, alpha 6, beta 2, beta 3, or delta. J. Neurosci. 26, 10315-10326 (2006).

24. He, Q. et al. Interneuron- and GABA(A) receptor-specific inhibitory synaptic plasticity in cerebellar Purkinje cells. Nat. Commun. 6, 7364 (2015).
25. Li, Q. et al. Prenatal immune challenge is an environmental risk factor for brain and behavior change relevant to schizophrenia: evidence from MRI in a mouse model. PLoS ONE 4, e6354 (2009).

26. Hui, K. M. et al. Anxiolytic effect of wogonin, a benzodiazepine receptor ligand isolated from Scutellaria baicalensis Georgi. Biochem. Pharmacol. 64, 1415-1424 (2002).

27. Peça, J. et al. Shank3 mutant mice display autistic-like behaviours and striatal dysfunction. Nature 472, 437-442 (2011).

28. Dellu, F., Contarino, A., Simon, H., Koob, G. F. \& Gold, L. H. Genetic differences in response to novelty and spatial memory using a two-trial recognition task in mice. Neurobiol. Learn. Mem. 73, 31-48 (2000).

29. $\mathrm{Xu}$, J., Zhu, Y., Contractor, A. \& Heinemann, S. F. mGluR5 has a critical role in inhibitory learning. J. Neurosci. 29, 3676-3684 (2009).

30. Park, S. J. et al. Toll-like receptor-2 deficiency induces schizophrenia-like behaviors in mice. Sci. Rep. 5, 8502 (2015).

31. Lu, X., Ross, B., Sanchez-Alavez, M., Zorrilla, E. P. \& Bartfai, T. Phenotypic analysis of GalR2 knockout mice in anxiety- and depression-related behavioral tests. Neuropeptides 42, 387-397 (2008).

32. Koo, J. W. \& Duman, R. S. IL-1beta is an essential mediator of the antineurogenic and anhedonic effects of stress. Proc. Natl Acad. Sci. USA 105 751-756 (2008).

33. Venit, E. L., Shepard, B. D. \& Seyfried, T. N. Oxygenation prevents sudden death in seizure-prone mice. Epilepsia 45, 993-996 (2004).

34. Ren, L. et al. GABA(A) receptor subtype selectivity underlying anxiolytic effect of 6-hydroxyflavone. Biochem. Pharmacol. 79, 1337-1344 (2010).

35. Pryor, W. A. On the detection of lipid peroxides in biological samples. Free Radic. Biol. Med. 7, 177-178 (1989).

36. Verde, $V$. et al. Use of $\mathrm{N}, \mathrm{N}$-dimethyl-p-phenylenediamine to evaluate the oxidative status of human plasma. Free Radic. Res. 36, 869-873 (2002).

37. Ge, Y. et al. Intrathecal infusion of hydrogen-rich normal saline attenuates neuropathic pain via inhibition of activation of spinal astrocytes and microglia in rats. PLOS ONE 9, e97436 (2014).

38. Richetto, J. et al. Behavioral effects of the benzodiazepine-positive allosteric modulator $\mathrm{SH}-053-2^{\prime} \mathrm{F}-\mathrm{S}-\mathrm{CH}_{3}$ in an immune-mediated neurodevelopmental disruption model. Int. J. Neuropsychopharmacol. 18, pyu055 (2015).

39. Götz, J. \& Ittner, L. M. Animal models of Alzheimer's disease and frontotemporal dementia. Nat. Rev. Neurosci. 9, 532-544 (2008).

40. Cascella, N. G., Schretlen, D. J. \& Sawa, A. Schizophrenia and epilepsy: is there a shared susceptibility? Neurosci. Res. 63, 227-235 (2009).

41. Chang, Y. T. et al. Bidirectional relation between schizophrenia and epilepsy: a population-based retrospective cohort study. Epilepsia 52, 2036-2042 (2011).

42. Edwards, J. G. Risperidone for schizophrenia. BMJ 308, 1311-1312 (1994).

43. Emiliani, F. E., Sedlak, T. W. \& Sawa, A. Oxidative stress and schizophrenia: recent breakthroughs from an old story. Curr. Opin. Psychiatry 27, 185-190 (2014).

44. Na, K. S., Jung, H. Y. \& Kim, Y. K. The role of pro-inflammatory cytokines in the neuroinflammation and neurogenesis of schizophrenia. Prog. Neuropsychopharmacol. Biol. Psychiatry 48, 277-286 (2014).

45. Radewicz, K., Garvey, L. J., Gentleman, S. M. \& Reynolds, R. Increase in HLA-DR immunoreactive microglia in frontal and temporal cortex of chronic schizophrenics. J. Neuropathol. Exp. Neurol. 59, 137-150 (2000).

46. Monji, A. et al. Neuroinflammation in schizophrenia especially focused on the role of microglia. Prog. Neuropsychopharmacol. Biol. Psychiatry 42, 115-121 (2013).

47. Laskaris, L. E. et al. Microglial activation and progressive brain changes in schizophrenia. Br. J. Pharmacol. 173, 666-680 (2016).

48. Donev, R. et al. Comorbidity of schizophrenia and adult attention-deficit hyperactivity disorder. World J. Biol. Psychiatry 12, 52-56 (2011).

49. Zhang, Z. J., \& Reynolds, G. P. A selective deficit in the relative density of parvalbumin-immunoreactive neurons in the hippocampus in schizophrenia. Schizophr. Res. 55, 1-10 (2002).

50. Allen, K. M., Fung, S. J. \& Weickert, C. S. Cell proliferation is reduced in the hippocampus in schizophrenia. Aust. N.Z. J. Psychiatry 50, 473-480 (2015).

51. Sohal, V. S., Zhang, F., Yizhar, O. \& Deisseroth, K. Parvalbumin neurons and gamma rhythms enhance cortical circuit performance. Nature 459, 698-702 (2009).

52. Gonzalez-Burgos, G. \& Lewis, D. A. NMDA receptor hypofunction, parvalbumin-positive neurons, and cortical gamma oscillations in schizophrenia. Schizophr. Bull. 38, 950-957 (2012).

53. Belelli, D. et al. Extrasynaptic GABAA receptors: form, pharmacology and function. J. Neurosci. 29, 12757-12763 (2009). 
54. Perea, G., Navarrete, M. \& Araque, A. Tripartite synapses: astrocytes process and control synaptic information. Trends Neurosci. 32, 421-431 (2009).

55. Lee, M., Schwab, C. \& McGeer, P. L. Astrocytes are GABAergic cells that modulate microglial activity. Glia 59, 152-165 (2011).

56. Losi, G., Mariotti, L., \& Carmignoto, G. GABAergic interneuron to astrocyte signalling: a neglected form of cell communication in the brain. Philos. Trans. $R$. Soc. Lond. BBiol. Sci 369, 20130609 (2014).

57. Cerbai, F. et al. The neuron-astrocyte-microglia triad in normal brain aging and in a model of neuroinflammation in the rat hippocampus. PLOS ONE 7 e45250 (2012).

58. Laursen, T. M., Agerbo, E. \& Pedersen, C. B. Bipolar disorder, schizoaffective disorder and schizophrenia overlap: a new comorbidity index. J. Clin. Psychiatry 70, 1432-1438 (2009).

59. Botta, P. et al. Regulating anxiety with extasynaptic inhibition. Nat. Neurosci. 18, 1493-1500 (2015)

60. Jones, C. A., Watson, D. J. \& Fone, K. C. Animal models of schizophrenia. Br. J. Pharmacol. 164, 1162-1194 (2011).

61. Spielewoy, C. et al. Behavioural disturbances associated with hyperdopaminergia in dopamine-transporter knockout mice. Behav. Pharmacol. 11, 279-290 (2000)

62. Ye, G. L. et al. in Transgenic and Mutant Tools to Model Brain Disorders (eds. Kalueff, A. V., Bergner, C. L.) 65-90 (Humana Press, New York, 2010).

63. Collinson, $\mathrm{N}$. et al. Enhanced learning and memory and altered GABAergic synaptic transmission in mice lacking the alpha 5 subunit of the $\mathrm{GABA}_{\mathrm{A}}$ receptor. J. Neurosci. 22, 5572-5580 (2002).

64. Duncan, G. E. et al. Deficits in sensorimotor gating and tests of social behavior in a genetic model of reduced NMDA receptor function. Behav. Brain Res. 153, 507-519 (2004)
65. Nakazawa, K. et al. GABAergic interneuron origin of schizophrenia pathophysiology. Neuropharmacology 62, 1574-1583 (2012).

66. Heckers, S. \& Konradi, C. GABAergic mechanisms of hippocampal hyperactivity in schizophrenia. Schizophr. Res. 167, 4-11 (2015).

67. Hörtnagl, $H$. et al. Patterns of mRNA and protein expression for 12 GABAA receptor subunits in the mouse brain. Neuroscience 236, 345-372 (2013).

68. Arundine, M. \& Tymianski, M. Molecular mechanisms of calcium-dependent neurodegeneration in excitotoxicity. Cell Calcium 34, 325-337 (2003).

69. Sekar, A. et al. Schizophrenia risk from complex variation of complement component 4. Nature 530, 177-183 (2016).

70. Guerriero, R. M., Giza, C. C. \& Rotenberg, A. Glutamate and GABA imbalance following traumatic brain injury. Curr. Neurol. Neurosci. Rep. 15, 27 (2015).

71. Eggers, C. \& Bunk, D. The long-term course of childhood-onset schizophrenia: a 42-year followup. Schizophr. Bull. 23, 105-117 (1997).

72. Laurie, D. J., Wisden, W. \& Seeburg, P. H. The distribution of thirteen GABAA receptor subunit mRNAs in the rat brain. III. Embryonic and postnatal development. J. Neurosci. 12, 4151-4172 (1992).

73. Harrison, P. J. Recent genetic findings in schizophrenia and their therapeutic relevance. J. Psychopharmacol. 29, 85-96 (2015).

74. Swerdlow, N. R. et al. Realistic expectations of prepulse inhibition in translational models for schizophrenia research. Psychopharmacology 199, 331-388 (2008).

75. George, M. et al. Newer antipsychotics and upcoming molecules for schizophrenia. Eur. J. Clin. Pharmacol. 69, 1497-1509 (2013).

76. Ballester, J. \& Frankel, B. A. Pharmacological advances in the treatment of schizophrenia. Am. J. Psychiatry 11, 5-8 (2016). 